\title{
Long Survival (Cure) to Cisplatin/Infusional 5-Flurouracil in Metastatic Squamous Cell Anal Cancer with Extensive Liver and Lung Metastases
}

\author{
Andrew Haydon ${ }^{1,2 *}$, Rebecca Tay ${ }^{1}$, Gabriel Mak ${ }^{1}$, Jeremy Shapiro ${ }^{1,2}$ \\ ${ }^{1}$ Department of Medical Oncology, Alfred Hospital, Melbourne, Australia \\ ${ }^{2}$ Monash University, Melbourne, Australia \\ Email: andrew.haydon@monash.edu
}

Received 18 February 2015; accepted 4 March 2015; published 10 March 2015

Copyright (C) 2015 by authors and Scientific Research Publishing Inc.

This work is licensed under the Creative Commons Attribution International License (CC BY). http://creativecommons.org/licenses/by/4.0/

\section{(c) (i) Open Access}

\begin{abstract}
Background: Metastatic anal Squamous Cell Carcinoma (SCC) is a relatively uncommon malignancy that is usually treated with palliative platinum based chemotherapy. Response rates are modest and complete responses are rare. Case Report: Here we report on a case of advanced SCC with the extensive visceral metastatic disease that has possibly been cured with chemotherapy alone. The patient has received 6 cycles of Cisplatin and 5FU and has remained in complete remission for over 7 years. Conclusion: This is the first report of a case of metastatic anal SCC that has been cured with chemotherapy and highlights the need to remain optimistic when treating patients with "palliative" chemotherapy.
\end{abstract}

\section{Keywords}

Metastatic Anal Squamous Cell carcinoma, Chemotherapy, Cure

\section{Background}

Anal cancer is rare, representing $<3 \%$ of all gastrointestinal system malignancies in the USA [1]. There is a close association between Human Papilloma Virus (HPV) infection and anal squamous cell carcinoma, with HIV infection well recognized to increase the risk of developing anal SCC. Other risk factors include chronic immu-

${ }^{*}$ Corresponding author.

How to cite this paper: Haydon, A., Tay, R., Mak, G. and Shapiro, J. (2015) Long Survival (Cure) to Cisplatin/Infusional 5Flurouracil in Metastatic Squamous Cell Anal Cancer with Extensive Liver and Lung Metastases. Case Reports in Clinical Medicine, 4, 73-76. http://dx.doi.org/10.4236/crcm.2015.43017 
nosuppression (not related to HIV infection), cigarette smoking and male homosexual intercourse. Most patients present with local symptoms. Tumours arising below the dentate line usually spread via lymphatics to the superficial inguinal and femoral lymph nodes, while internal pelvic nodal metastases arise from more proximal primaries. Distant metastatic spread at diagnosis is uncommon. Patients with localized disease are usually managed with definitive, curative-intent, chemo-radiation with very good long-term outcome, with surgery reserved for local failure. In this group, tumour human papilloma virus status, demonstrated by positive p16 immunohistochemical staining, equates to a significantly better prognosis [2].

However, metastatic spread to distant organs is associated with a poor prognosis and median survival is around 12 months [3] [4]. In metastatic disease, standard first-line chemotherapy is cisplatin and 5-flurouracil [5]-[7]. Reported response rates are $50 \%$, with complete responses rarely seen [7].

There is no standard second line therapy with the rarity of this tumour precluding the completion of large randomised studies. Agents with reported salvage activity in single institute series and phase II trials include mitomycin/doxorubincin/cisplatin [3], as well as single agent activity with cetuximab [8] [9], carboplatin [10] and irinotecan [11], however clinical benefit remains modest. Occasional reports of prolonged survival following chemotherapy in metastatic anal cancer have been documented in patients who have liver-only metastases and are able to undergo resection of residual hepatic disease [5].

This case report highlights a patient who, despite having widespread metastatic disease, appears to have been cured with cisplatin and 5FU chemotherapy.

\section{Case Report}

A 70-year-old HIV negative Caucasian female, with no significant past medical history, presented with a $2.5 \mathrm{~cm}$ muscle invasive anal cancer, involving pubo-rectalis and anal sphincter on endoscopic ultrasound. Examination was otherwise normal. Computerised tomography (CT) of the chest, abdomen and pelvis demonstrated widespread bilobar liver metastases ( $>50 \%$ liver volume. Figure 1(a)), as well as multiple pulmonary metastases, and abdominal nodal spread. Biopsy of the primary lesion demonstrated an anal squamous cell carcinoma with basaloid differentiation (Figure 2(a)). Immunohistochemical expression of p16 was strongly positive (Figure 2(b)), indicating the presence of human papilloma virus (HPV). Immunoperoxidase stains for chromogranin and synaptophysin were negative. Baseline biochemical investigations, including tumour markers were all within normal limits.

Six cycles of 3-weekly cisplatin (75 mg/m² Day 1) and infusional 5-flurouracil (5-FU) $4000 \mathrm{mg} / \mathrm{m}^{2}$ over 96 hours was administered with palliative intent. Rapid clinical improvement ensued, with marked cancer regression on clinical examination and CT after 3 cycles. Clinical examination and CT scan had normalised after 6 cycles and chemotherapy was ceased without any local treatment to the primary tumour. Seven years later, the patient remains in ongoing complete clinical and radiological remission (Figure 1(b)).

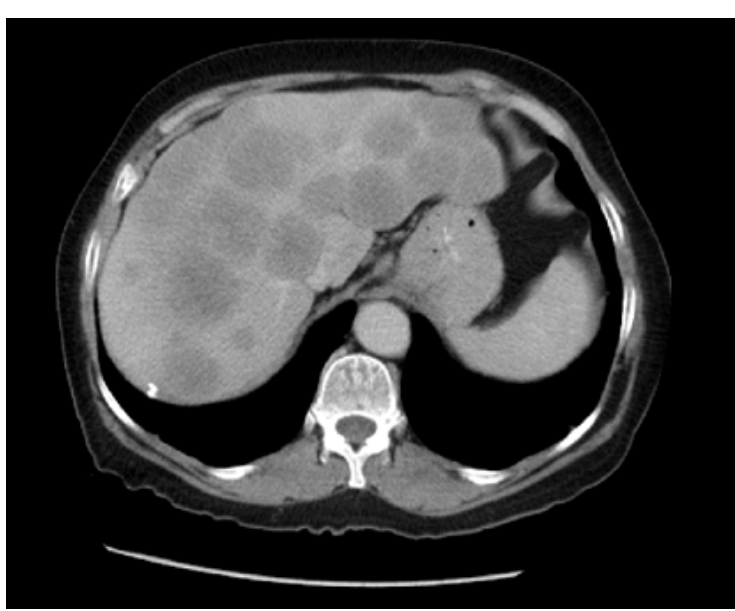

(a)

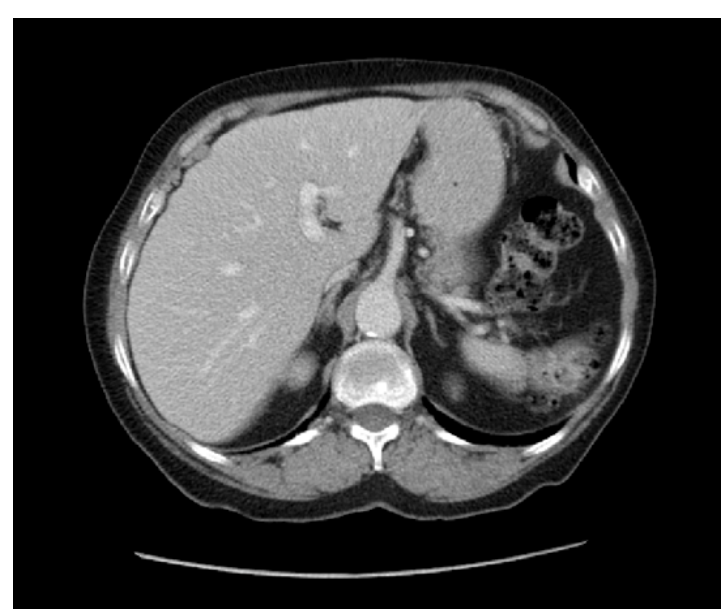

(b)

Figure 1. CT axial view of abdomen demonstrated multiple liver metastases at time of diagnosis (a), and an ongoing complete remission at 7 yrs (b). 


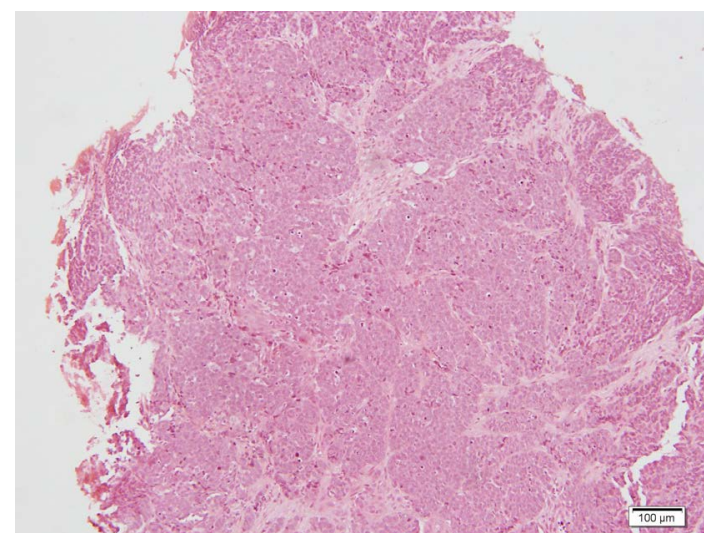

(a)

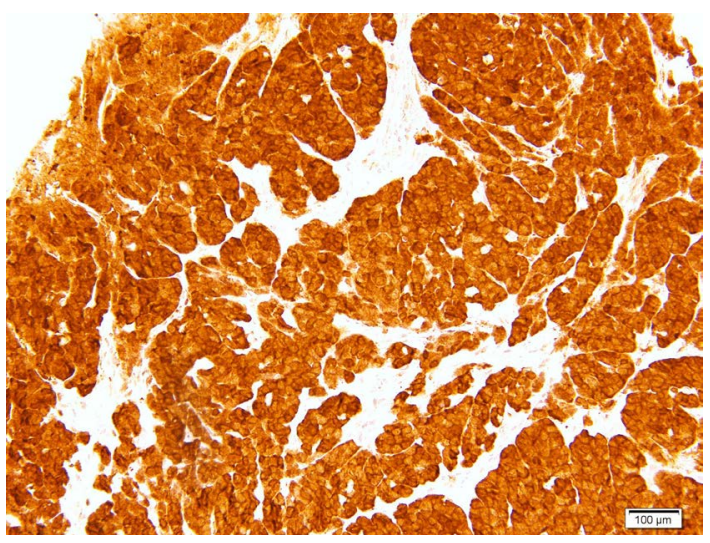

(b)

Figure 2. Histopathology of rectal biopsy. H \& E section (a); immunohistochemistry for p16 showing strong immunoreactivity (b).

\section{Conclusions}

This case report illustrates a complete response to cisplatin/infusional 5FU chemotherapy despite widespread metastatic disease including very extensive liver involvement, and sustained and ongoing complete remission (without surgery), beyond seven years of follow-up.

Following the remarkable response to chemotherapy seen in our patient, we went back to the original histology and requested that p16 immunohistochemistry should be performed. The strong positive staining (Figure 2(b)) and excellent response to chemotherapy are consistent with other reports suggesting improved outcome with HPV associated tumours [2]. Currently there is not enough evidence to recommend the routine use of p16 immunohistochemistry to guide treatment decisions in metastatic SCC of the anus, however, given this case, perhaps oncologists can be slightly more optimistic when counseling patients with p16 positive metastatic anal SCC.

To our knowledge, this is the 1st report of a potential cure of metastatic anal SCC with systemic chemotherapy as the sole treatment modality.

Written informed consent to publish this case report was obtained from our patient.

\section{References}

[1] Siegel, R.L., Miller, K.D. and Jemal, A. (2015) Cancer Statistics. CA: A Cancer Journal for Clinicians, 65, 5-29.

[2] Yhim, H., Lee, R.N., Song, E.K., Kwak, J.Y., Lee, S.T., et al. (2011) The Prognostic Significance of Tumor Human Papillomavirus Status for Patients with Anal Squamous Cell Carcinoma Treated with Combined Chemoradiotherapy. International Journal of Cancer, 129, 1752-1760. http://dx.doi.org/10.1002/ijc.25825

[3] Jhawer, M., Mani, S., Lefkopoulous, M., Hahn, R.G., Harris, J., et al. (2006) Phase II Study of Mitomycin-C, Adriamycin, Cisplatin (MAP) and Bleomycin-CCNU in Patients with Advanced Cancer of the Anal Canal: An Eastern Cooperative Oncology Group Study E7282. Investigational New Drugs, 24, 447-454. http://dx.doi.org/10.1007/s10637-006-7667-x

[4] Tanum, G. (1993) Treatment of Relapsing Anal Carcinoma. Acta Oncologica, 32, 33-35. http://dx.doi.org/10.3109/02841869309083882

[5] Ajani, J.A., Carrasco, C.H., Jackson, D.E. and Wallace, S. (1989) Combination of Cisplatin Plus Fluoropyrimidine Chemotherapy Effective against Liver Metastases from Carcinoma of the Anal Canal. The American Journal of Medicine, 87, 221-224. http://dx.doi.org/10.1016/S0002-9343(89)80702-8

[6] Khater, R., Frenay, M., Bourry, J., Milano, G. and Namer, M. (1986) Cisplatin Plus 5-Fluorouracil in the Treatment of Metastatic anal Squamous Cell Carcinoma: A Report of Two Cases. Cancer Treatment Reports, 70, 1345-1346.

[7] Faivre, C., Rougier, P., Ducreux, M., Mitry, E., Lusinchi, A., et al. (1999) 5-Fluorouracile and Cisplatinum Combination Chemotherapy for Metastatic Squamous-Cell Anal Cancer. Bull Cancer, 86, 861-865.

[8] Saif, M., Kontny, E., Syrigos, K.N. and Shahrokni, A. (2011) The Role of EGFR Inhibitors in the Treatment of Metastatic Anal Canal Carcinoma: A Case Series. Journal of Oncology, 125467. 
[9] Lukan, N., Ströbel, P., Willer, A., Kripp, M., Dinter, D., et al. (2009) Cetuximab-Based Treatment of Metastatic Anal Cancer: Correlation of Response with KRAS Mutational Status. Oncology, 77, 293-299.

[10] Evan, T., Mansi, J. and Glees, J. (1993) Response of Metastatic Anal Carcinoma to Single Agent Carboplatin. Clinical Oncology, 5, 57-58. http://dx.doi.org/10.1016/S0936-6555(05)80699-5

[11] Grifaichi, F., Padovani, A., Pomeo, F., Trinca, C., Moscetti, L. and Cortesi, E. (2001) Response of Metastatic Epidermoid Anal Cancer to Single Agent Irinotecan: A Case Report. Tumori, 87, 58-59. 